\title{
Sistema de vigilancia epidemiológica para el paciente diabético; el uso de tecnología computacional en la calidad de la atención médica
}

\author{
Ramón Alberto Rascón-Pacheco, M.C ., M. en C., ${ }^{(1)}$ Manuel Alberto Santillana-Macedo, M.C ., M. en C., (2) \\ María Elena Romero-Arredondo, Ing. en Sist., (1) Blanca Margarita Rivera-Icedo, Psic., ${ }^{(1)}$ \\ José Alfredo Romero-C ancio, M.C., ${ }^{(2)}$ Alex Iván C ota-Rembau, Ing. en Sist. ${ }^{(3)}$
}

\begin{abstract}
Rascón-Pacheco RA, Santillana-Macedo MA, Romero-Arredondo ME, Rivera-Icedo BM, Romero-Cancio JA, Cota-Rembau AI. Sistema de vigilancia epidemiológica para el paciente diabético; el uso de tecnología computacional en la calidad de la atención médica. Salud Publica Mex 2000;42:324-332.
\end{abstract}

\section{Resumen}

Objetivo. Presentar un instrumento que permita llevar a cabo la vigilancia epidemiológica y evaluar la calidad de la atención a la diabetes mellitus en el primer nivel de atención. Material y métodos. Este estudio se realizó del 1 de enero de 1998 al 30 de junio de 1999, en la Unidad de Investigación Epidemiológica y en Servicios de Salud, del Instituto Mexicano del Seguro Social (IMSS), en Hermosillo, Sonora. Se diseñó un formato único de reporte compuesto por los diferentes elementos que integran el sistema para la atención del paciente diabético en una Unidad de Medicina Familiar del IMSS. Resultados. Se desarrolló e implementó un paquete de computación (software) que permite la captura de los datos de dicho formato y la generación de reportes, de tipo individual y grupal, sobre el cumplimiento de citas, antecedentes personales patológicos y no patológicos, evolución de signos y síntomas, exámenes de laboratorio y el manejo terapéutico. Conclusiones La sistematización de datos en forma electrónica favorece la obtención de información confiable y permanente para la vigilancia de los procesos en la atención integral del paciente diabético.

Palabras clave: sistemas de información; servicios de salud; vigilancia epidemiológica; diabetes mellitus no insulino-dependiente; México

\author{
Rascón-Pacheco RA, Santillana-Macedo MA, \\ Romero-Arredondo ME, Rivera-Icedo BM, \\ Romero-Cancio JA, Cota-Rembau AI. \\ Epidemiologic surveillance \\ of diabetic patients; use of computer technology \\ in the quality of medical care. \\ Salud Publica Mex 2000;42:324-332.
}

\begin{abstract}
A bstract
Objective.To describe an instrument for epidemio lo gic surveillance of diabetes mellitus and evaluating the quality of care in primary health care. Material and methods. The study was conducted from January 1,1998, to June 30,1999, at the Epidemiology and Health Services Research U nit of the Mexican Institute for So cial Security (IMSS), in HermosiIlo, Sonora. A single data collection form was designed, which contains items included in the diabetes care clinic of the Unit of Family Medicine of the Mexican Institute of Social Security. Results. We developed and implemented a software program to enter data from the paper format and to generate individual and group reports on attendance to scheduled medical visits, medical history, evolution of signs and symptoms, laboratory tests, and medication. Conclusions Electronic data systems allow the availability of reliable and continuing information for surveillance of the comprehensive care of the diabetic patient.
\end{abstract}

Key words: information systems; health services; epidemio lo gic surveillance; diabetes mellitus, non-insulin-dependent; Mexico

Registro del proyecto en el IMSS: 98-517-001.

(1) Unidad de Investigación Epidemiológica y en Servicios de Salud. Instituto Mexicano del Seguro Social (IMSS), Hermosillo, Sonora, México.

(2) Unidad de Medicina Familiar N 0. 37, IMSS, Hermosillo, Sonora, México.

(3) Instituto Tecnológico de Hermosillo, Sonora, México.

Fecha de recibido: 20 de enero de 2000 - Fecha de aprobado: 22 de mayo de 2000

Solicitud de sobretiros: Dr. Ramón Alberto Rascón Pacheco. Unidad de Investigación Epidemiológica y en Servicios de Salud-Hermosillo, Instituto Mexicano del Seguro Social. Hospital General Regional N 0.2, Planta baja, calle Juárez y Seguro Social, colonia Modelo, 83150 Hermosillo, Sonora, México.

Correo electrónico: uiesshmo@ prodigy.net.mx 
L a calidad de la atención a la salud la define Donabedian como "el grado en el que los medios más deseables se utilizan para alcanzar las mayores mejoras posibles en salud". ${ }^{1}$ Además, la atención a la salud debe considerar la solución de una necesidad mediante un proceso sistematizado de toma de decisiones cuyo resultado es la modificación de esa necesidad dentro del ámbito de una organización y de factores socioculturales. ${ }^{2}$

En este sentido, medir e informar el estado de salud de una determinada población es crucial para cualquiera que esté preocupado por brindar servicios con calidad a la población. Para garantizar la calidad, según el propio Donabedian, se debe contar con dos elementos inseparables, a saber: el diseño del sistema y la monitoría del desempeño. ${ }^{1}$ Un sistema de vigilancia epidemiológica permite integrar estos dos elementos de tal forma que se pueda obtener información oportuna que facilite tomar decisiones o hacer recomendaciones a corto, mediano o largo plazo, sobre bases objetivas y científicas, con el propósito de prevenir o controlar un problema de salud, como la diabetes mellitus tipo 2 (DM2), ${ }^{3}$ reconocido por su alta prevalencia y su consecuente repercusión en la utilización de servicios de salud.

La DM2, especialmente cuando no está controlada, puede representar una pesada carga económica para el individuo y la sociedad. Así, dependiendo del país, los cálculos indican que la diabetes puede representar entre 5 y $14 \%$ de los gastos de asistencia sanitaria. ${ }^{4}$ En el Instituto Mexicano del Seguro Social (IMSS) el gasto promedio anual de la atención a los enfermos que padecen DM2 superó los 2000 millones de pesos durante el periodo comprendido entre 1992 y $1997 .{ }^{5}$ Sin embargo, muchos de estos gastos en atención a la DM2 se pueden reducir, aplazar y, en ciertos casos, prevenir. Esto exige de una serie de acciones y decisiones coordinadas donde se incluya la alimentación, ejercicio físico, cambios en los hábitos del estilo de vida y, de requerirse, la medicación hipoglicemiante.

Actualmente, en varias de las instituciones proveedoras de servicios de salud se generan datos que requieren, para el análisis eficiente, de una sistematización sobre todo en los ámbitos locales, pero de gran demanda de servicios de salud, como lo son las Unidades de Medicina Familiar (UMF). ${ }^{6}$ Sin embargo, se tiene la suposición de que para mejorar los sistemas de información se requiere hacer un gasto excesivo en relación con los beneficios que proporciona. ${ }^{7}$ Adicionalmente, en la actualidad la acelerada dinámica en los procesos y necesidades de información da como resultado que ésta en muchas ocasiones se obtenga a muy elevado costo, tanto en lo económico como en re- cursos humanos y materiales, sin que se cumpla con el objetivo de dar fundamento apropiado a la toma de decisiones.

Este supuesto no necesariamente es así, ya que hoy en día es posible combinar los elementos de un sistema de vigilancia epidemiológica con los de un sistema computarizado que permita mejorar la calidad de la información, a bajo costo, con beneficios tanto para el paciente como para la institución proveedora de los servicios de salud. En el marco de la normatividad para el manejo y control de los pacientes con diabetes mellitus, en las UMF existen los elementos necesarios para constituir un sistema de vigilancia epidemiológica. Empero, estos elementos, a la fecha, se han desarrollado con un procedimiento manual y operativamente desarticulado, lo que dificulta convertir los datos, a pesar de que se producen y registran enormes cantidades de ellos, en información que esté a disposición del personal indicado, en el momento preciso y en un formato simple. ${ }^{8}$

En este sentido, se propone la sistematización electrónica de la información recabada en los datos del expediente clínico para coordinar los esfuerzos del sistema de atención por el médico familiar del IMSS en el control de este padecimiento. Puntualmente, el desempeño del médico familiar se caracteriza por el desarrollo de una serie de actividades que son registradas manualmente, algunas de las cuales son registradas en sistemas automatizados institucionales a los que el personal que las generó no tiene acceso directo sino, más bien, por medio de los directivos de los que depende. No obstante, esta información no cuenta con los suficientes elementos que le permitan conocer la epidemiología de los motivos de consulta y las características propias de la misma.

El objetivo de este trabajo es presentar a la comunidad científica y asistencial un instrumento para evaluar la calidad de la atención y vigilancia epidemiológica de la diabetes mellitus en las UMF. Se indican los pasos que se siguieron en la elaboración de un sistema, así como los recursos que éste aporta para la evaluación individual y grupal de la aplicación del programa institucional para el control de los pacientes diabéticos.

\section{Material y métodos}

Este estudio se realizó del 1 de enero de 1998 al 30 de junio de 1999. En esta primera etapa, el equipo de trabajo de la Unidad de Investigación Epidemiológica y en Servicios de Salud (UIESS) se dio a la tarea de identificar los procesos que integran el sistema para la atención del paciente diabético en una UMF; asimis- 
mo, los datos que se utilizan en cada uno de los procesos, los que se almacenan y que entran y salen del sistema.

Posteriormente, se diseñó un formato que permite capturar los datos necesarios para integrar los diferentes procesos. Además, este formato atiende a los lineamientos del Programa Institucional para la Vigilancia, Prevención y Control de la Diabetes Mellitus del IMSS 5 y los criterios de diagnóstico, control y seguimiento de la American Diabetes Association (ADA). ${ }^{9}$

En el siguiente paso se ideó la estructura de una base de datos electrónica que contiene seis tablas, con el número de afiliación como campo llave. Esta base se desarrolló en el lenguaje Visual FoxPro versión 6.0, que permite la captura (entrada) de los datos de identificación del paciente diabético, cumplimiento de citas, antecedentes personales patológicos y no patológicos, evolución de signos y síntomas, exámenes de laboratorio y manejo del caso.

Asimismo, se diseñaron reportes (salidas) que permiten localizar a cada uno de los pacientes, evaluar la inasistencia a la consulta, presencia de daño renal incipiente, daño de la retina y pacientes con franco descontrol de las glicemias. Por último, se diseñó una aplicación que permite respaldar cada una de las tablas con el fin de explotar al máximo los datos generados por el Sistema de Vigilancia Epidemiológica para el Paciente Diabético (SVEPD).

Con el propósito de confirmar el buen funcionamiento del sistema se capturaron los datos del menú principal y el apego a citas de la totalidad de los pacientes diabéticos del turno matutino de una UMF de la Delegación Sonora del IMSS. Una vez realizado este censo se tomó una muestra de 10 pacientes por cada consultorio con el propósito de obtener de los expedientes clínicos los datos necesarios para llenar los diferentes módulos que conforman el SVEPD.

\section{Resultados}

Como resultado del primer análisis se creó un formato único que incorpora los datos generados de la tarjeta denominada Vigilancia y control (VYC), que es llenada por las asistentes médicas. También capta los datos de los exámenes de laboratorio y los aspectos clínicos del paciente que son registrados en el expediente clínico y que es llenado por el médico familiar, en un formato denominado Control del paciente diabético (MF5), en cada consulta. Además, el equipo de trabajo social de la UMF lleva una bitácora de las diferentes actividades de capacitación de los pacientes con DM2 (figura 1).
Para capturar los datos de este formato se diseñó una base de datos conformada por seis tablas y sus respectivas pantallas de captura:

Vigilancia y control. Esta tabla funciona como eje del SVEPD y permite almacenar los datos de identificación de los pacientes, tales como nombre, número de afiliación, consultorio, turno de atención, teléfono, domicilio y señales de localización. Además, se captura la fecha de asistencia del paciente a las diversas actividades educativas que son preparadas por el personal de la UMF.

Por otro lado, en esta pantalla existen en la parte inferior seis íconos que permiten pasar a cinco pantallas de captura y una de reporte (figura 2), las cuales se describen a continuación:

- Citas. Aquí se registra la fecha de la cita para su control. Además, en este subsistema es posible consignar la asistencia a la consulta (o inasistencia) de los pacientes.

- Antecedentes personales. Esta tabla permite capturar los datos de los antecedentes personales patológicos y no patológicos de los pacientes; además, características demográficas como edad, sexo, escolaridad y ocupación.

- Signos y síntomas. En este subsistema se integran los datos correspondientes a los signos y síntomas como son: presencia de polifagia, poliuria, polidipsia, sensibilidad y pulsos en los pies, peso, índice de masa corporal y tensión arterial. Del mismo modo, el médico familiar anota el informe del examen de fondo de ojo, el cual, según el Programa Institucional, debe realizarse en un segundo nivel de atención; asimismo, en este apartado se captura si el paciente presentó alguna crisis aguda como cetoacidosis o hipoglucemia.

- Exámenes de laboratorio. Aquí se capturan los exámenes de laboratorio tanto de sangre como de orina necesarios para vigilar la evolución del padecimiento como son hemoglobina glucosilada, glucosa en ayunas, perfil de lípidos, examen general de orina, microalbuminuria, etcétera.

- Manejo del caso. En esta sección del sistema se introducen los datos correspondientes al manejo farmacológico, evolución de la condición física (ejercicio) y régimen dietético.

Una vez capturados los datos de los pacientes diabéticos el sistema es capaz de generar, con la periodicidad que se requiera, cinco reportes a manera de listado con información sobre: 


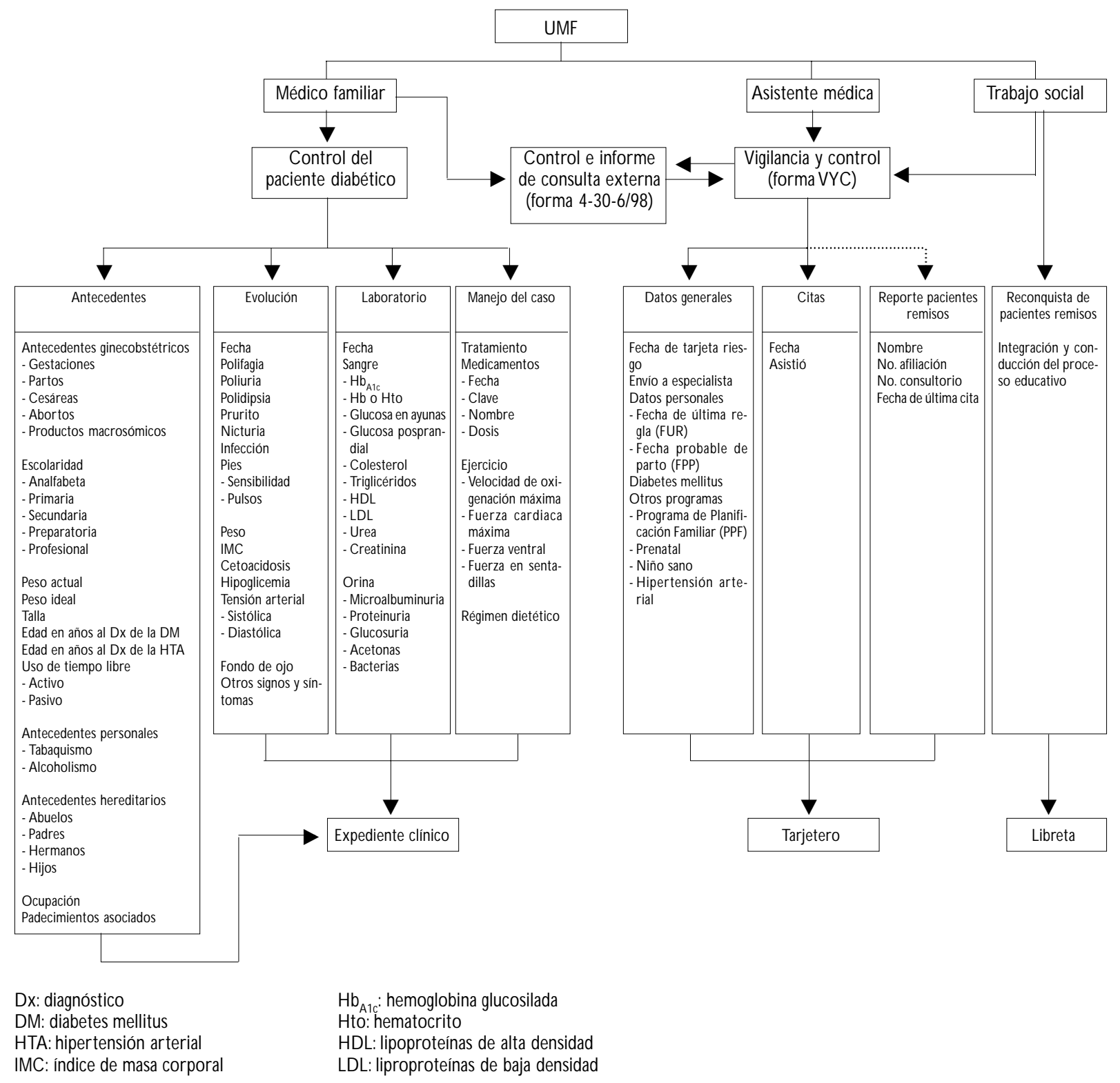

Figura 1. Elementos que forman el subsistema de atención del paciente diabético en las Unidades de Medicina Familiar. Unidad de Investigación Epidemiológica y en Servicios de Salud, Instituto Mexicano del Seguro Social. Hermosillo, Sonora, México, 1998-1999

1) Datos generales del total de pacientes

2) Pacientes que en ese momento tienen 90 o más días sin acudir a la consulta (figura 3 )

3) Presencia de microalbuminuria

4) Pacientes que en ese momento tienen más de un año sin acudir al oftalmólogo

5) Promedio de glucosa en ayunas mayor o igual a $140 \mathrm{mg} / 100 \mathrm{ml}$.
Además de los informes arriba mencionados, este sistema genera en código ASCII, un archivo por cada tabla, lo que permite hacer un análisis de los diversos contenidos de la base de datos del SVEPD. Así, y a manera de ejemplo, a continuación se presentan algunos de los tantos análisis que se pueden realizar: en la figura 4 se muestra la distribución del número de consultas durante el año de 1998 de los pacientes diabé- 


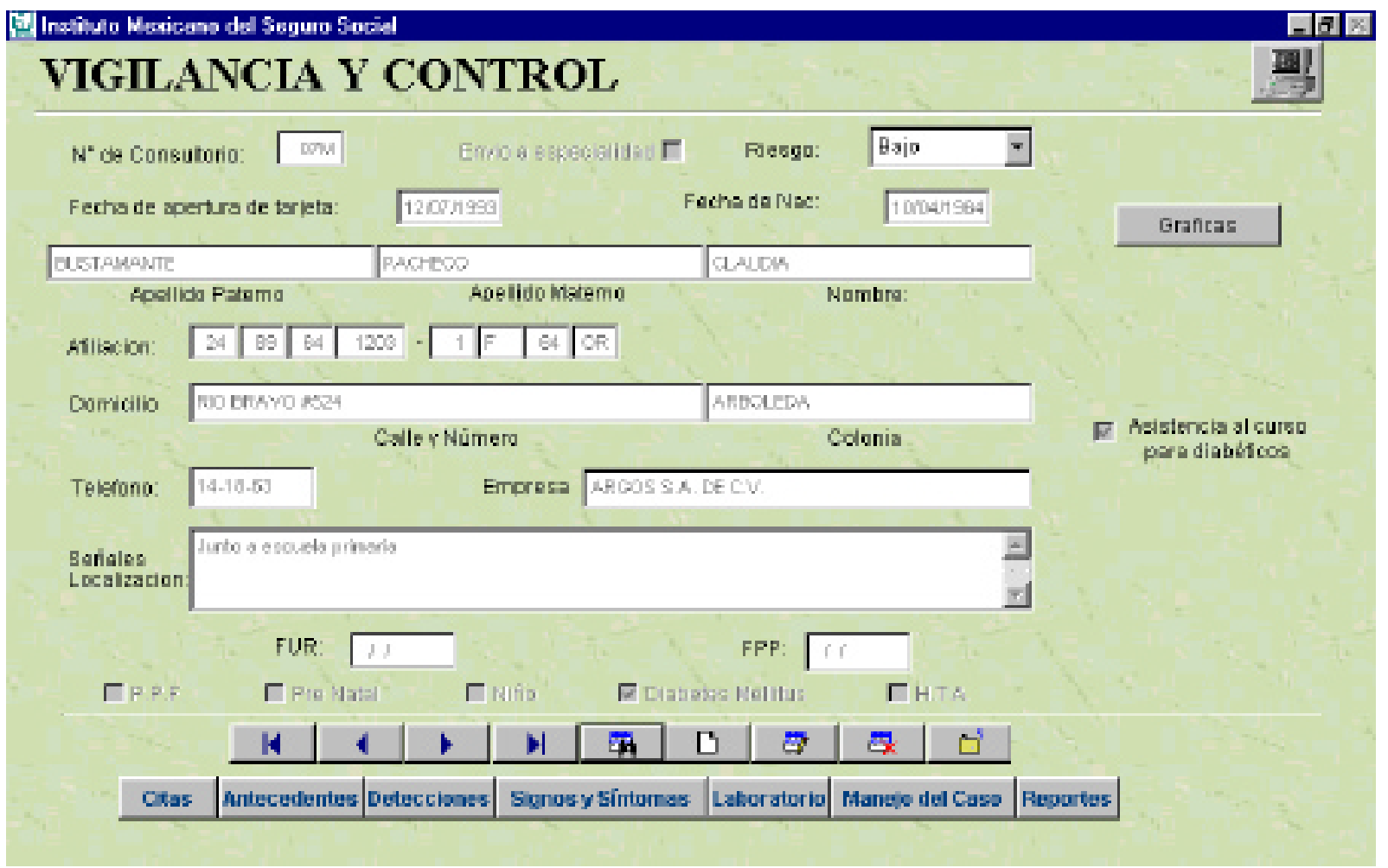

Figura 2. Pantalla de captura de datos de la tarjeta de vigilancia y control y menú principal. Unidad de Investigación Epidemiológica y en Servicios de Salud, In stituto Mexicano del Seguro Social. Hermosillo, SONORA, MÉxico, 1998-1999

ticos, según el consultorio; en la figura 5 se integran, para cada uno de los pacientes, tres aspectos fundamentales del control de la diabetes como: índice de masa corporal (IMC); presión sistólica y diastólica, y los niveles de glucosa en ayunas entre el 1 de enero y el 30 de julio de 1999.

\section{Discusión}

El estado del arte sobre la fisiopatología y manejo del paciente con DM2 es amplio; así, se sabe que el curso de esta afección es la causa de un gran número de complicaciones que generan un serio trastorno en la calidad de vida de los pacientes por su gravedad y cronicidad. En efecto, existe un consenso creciente en el sentido de que las complicaciones crónicas de la diabetes son secundarias al trastorno metabólico. ${ }^{10}$ Hasta que la curación sea una realidad, la meta de la terapéutica en la diabetes mellitus es obtener el mejor con- trol metabólico posible que, en términos prácticos, se reduce a que el paciente logre conservar sus cifras de glucosa lo más cercanas posible a lo normal. ${ }^{11}$

La utilidad de los programas sistematizados de vigilancia epidemiológica ha sido demostrada. De hecho, el Programa Nacional de Vacunación ${ }^{12}$ (Provac) es una muestra exitosa de la coordinación entre las acciones de salud y el control sistematizado de un programa computarizado. Vigente en la actualidad, el Provac consta, en una de sus fases, del registro electrónico de información de los menores de cinco años de edad respecto de su estado vacunal y datos de localización, y entre sus productos se encuentran listados de niños con esquemas de vacunación incompletos mediante los cuales las unidades operativas llevan un control de los menores de cinco años de su área de responsabilidad. Esto redundó, como es conocido, en un beneficio a la población mexicana al incrementar sustancialmente la proporción de población infantil 


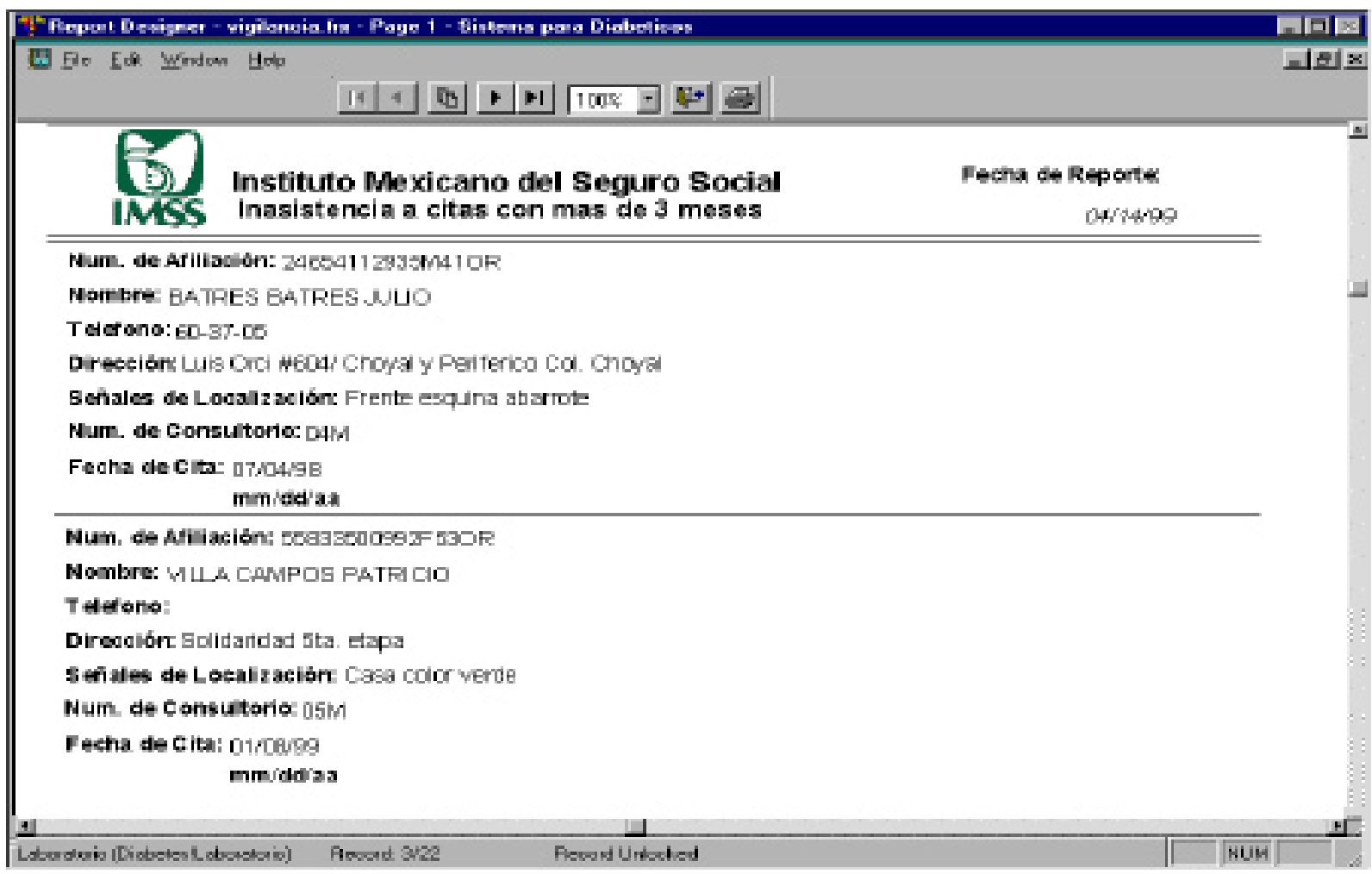

Figura 3. Reporte que emite el Sistema de Vigilancia Epidemiológica del Paciente Diabético. Unidad de Investigación Epidemiológica y en Servicios de Salud, In stituto Mexicano del Seguro Social. Hermosillo, SonoRA, MÉxico, 1998-1999

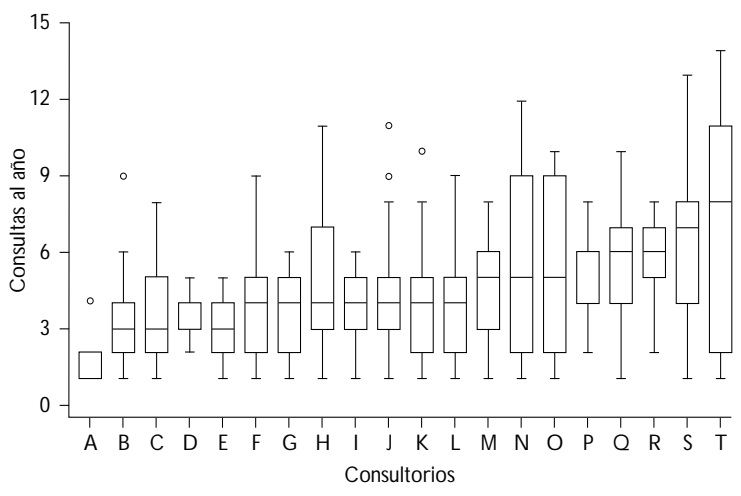

Con el propósito de mantener la confidencialidad se ordenó según la mediana y se asignó de forma alfabética el nombre del consultorio

Figura 4. Distribución del número de Visitas al año DURANTE 1998, SEGÚN CONSULTORIO.* UNIDAD DE INvestigación Epidemiológica y en Servicios de Salud, Instituto Mexicano del Seguro Social. Hermosillo, SONORA, México, 1998-1999 vacunada, al disminuir la prevalencia de enfermedades prevenibles por vacunación como poliomielitis, sarampión y tétanos neonatal. ${ }^{13}$

Dentro del marco de la evaluación y medición de la calidad de la atención, un sistema de vigilancia epidemiológica nos permite identificar oportunamente los problemas de una enfermedad y atenderlos con precisión y efectividad. ${ }^{14}$ En la actualidad ya se encuentran documentados los sistemas de vigilancia epidemiológica con la colaboración de sistemas computacionales que permiten acelerar la toma de decisiones efectivas y oportunas para pacientes y los servicios de salud. ${ }^{15}$ En efecto, el estudio del Hospital de Veteranos con Pacientes Diabéticos de Nueva Jersey demostró su utilidad tanto en el buen control y seguimiento de los pacientes como en el monitoreo del manejo farmacológico, de laboratorio y de los costos de la atención. ${ }^{15}$ En este sentido, el diseño de un sistema de vigilancia epidemiológica para el paciente diabético en unidades de medicina familiar mexicanas puede resultar de gran ayuda. 

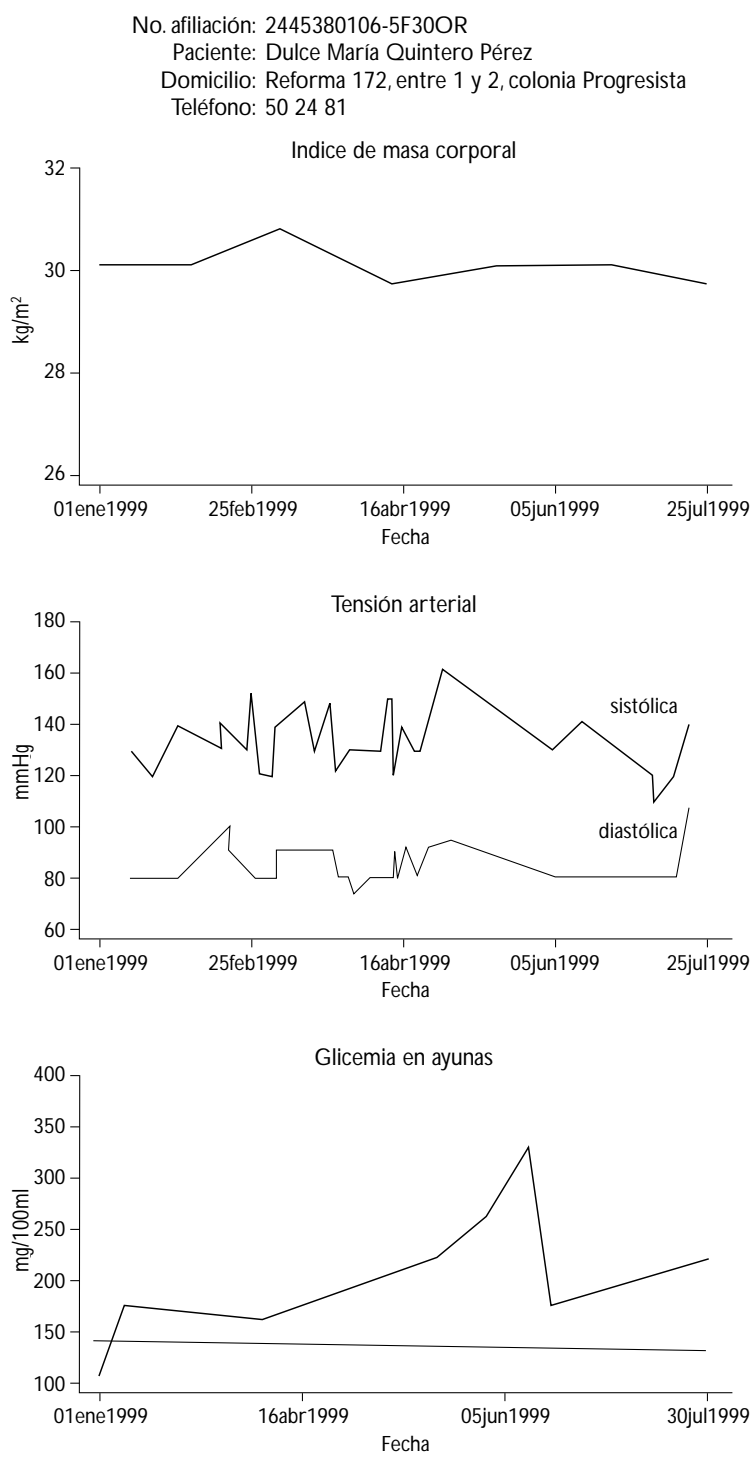

Figura 5. EJEMPLO de tRes INDICAdores PARA EL CONTROL INDIVIDUAL DE UN PACIENTE DIABÉTICO. UNIDAD DE Investigación Epidemiológica y en Servicios de Salud, Instituto Mexicano del Seguro Social. HerMOSILLO, SONORA, MéXICO, 1998-1999

El sistema que ahora se presenta tiene varios antecedentes; de hecho, el manejo del paciente diabético tipo 2 conforma ya un programa integral del IMSS $^{5}$ al que se puede articular de una forma eficiente. Es decir, no es necesario restructurar la normatividad o modificar abruptamente la propuesta integral sino complementarse con un apoyo tecnológico. Ciertamen- te, una característica clave de este sistema de vigilancia epidemiológica para el paciente diabético es que funciona como un integrador entre las acciones de prevención, detección oportuna de factores de riesgo y posibles complicaciones con las actividades de control médico, nutricional, de actividad física y cambios en el estilo de vida.

Además, se debe considerar como un sistema de control de una cohorte de pacientes diabéticos de la comunidad donde esté ubicada la unidad de medicina familiar. Sin embargo, es importante destacar que la perspectiva integradora de este sistema de vigilancia epidemiológica no suple las actividades propias de la atención primaria a la salud, a saber: la educación para la salud, el desarrollo de actividades promotoras de la salud en la comunidad, detección oportuna por pruebas de tamizaje, exámenes diagnósticos sensibles y específicos, atención integral por medicina familiar y envío oportuno -en los casos que lo requierana subespecialidades para evitar o controlar las posibles complicaciones.

Lo que sí puede hacer este sistema es integrar una buena parte de la información de estas actividades mediante un sistema computacional que, como señaló Donabedian, ${ }^{1}$ monitorea el desempeño de un programa de atención. La realidad virtual no suple a la realidad concreta en salud. La perspectiva de los autores es que un sistema computarizado de vigilancia epidemiológica es útil, pero su utilización real depende de integrar, de hecho, todas las actividades poco conectadas actualmente de prevención, atención y rehabilitación.

Si se atiende a las salidas del sistema es posible confeccionar tres áreas de estudio. Una de ellas es la información censal de los pacientes diabéticos; la segunda, la del control de la evolución de los pacientes, y la tercera, de la calidad de la atención brindada en esa unidad médica. Al conformarse como un sistema organizado, el nivel de desagregación puede hacerse por turno, consultorio o por paciente. De este modo, fue posible determinar el número de diabéticos en el turno matutino de la UMF de estudio, que fue en total de 1641 diabéticos mayores de 20 años y que el promedio de diabéticos por consultorio registrado fue de 80 pacientes.

Simultáneamente, el nivel de agregación permite conocer las características poblacionales de todos los diabéticos, así como hacer comparaciones entre grupos de edad, laborales, por evolución, por resultados de laboratorio, etcétera. Esta es la riqueza que genera el poder cruzar la información del sistema entre sí, de acuerdo con intereses diversos. 
Por otro lado, con las salidas del SVEPD es posible determinar la proporción de los sujetos que tienen más de tres meses de no asistir a una consulta de control. En la UMF de estudio se encontró que, de acuerdo con el consultorio, esta proporción varía desde 7.7 hasta $38 \%$. Al no ser uniforme el apego a las citas, esto obliga a los directivos a buscar el origen con el propósito de diseñar un plan que permita corregir cualquier desviación. Además de utilizar este sistema como un instrumento de diagnóstico, puede servir como una herramienta para verificar el cumplimiento de dicho plan. En la figura 4 se puede observar que el SVEPD genera datos que permiten estimar dónde se encuentran los pacientes, quiénes se presentan menos asiduamente a su consulta y, a la vez, estimar si esta asiduidad está relacionada con su control metabólico, IMC o, incluso, manejo de medicamentos. Estos análisis pueden ser tan detallados como se necesiten.

En cuanto a la evaluación de la calidad de la atención, los sistemas computacionales sirven tanto para evaluar la evolución individual y grupal de los pacientes diabéticos como para valorar la calidad de la información generada. Por ejemplo, el estudio de Hofer señala que, además de las ventajas de vigilar la evolución de los pacientes diabéticos por medio de los registros clínicos y de laboratorio, se puede evaluar la calidad de la atención en los registros de los expedientes de los médicos. ${ }^{16}$ Así, el sistema de vigilancia epidemiológica del paciente diabético, que se presenta para una UMF del IMSS, permite evaluar la consistencia de los datos incorporados al expediente de los antecedentes personales, la evolución del padecimiento y los exámenes de laboratorio. Estos tres aspectos valoran de una forma indirecta la operatividad del sistema médico familiar en la atención que brinda a la DM2.

Primordialmente, la práctica del médico familiar se limita a proporcionar atención asistencial, matizada con un enfoque preventivo respecto de ciertas patologías o programas institucionales a la población derechohabiente adscrita a su consultorio. Esta atención se encuentra, además, acompañada de algunas acciones de promoción de la salud con un enfoque en los programas institucionales prioritarios. ${ }^{17} \mathrm{La}$ posibilidad de contar con un sistema automatizado, particularmente el de la vigilancia epidemiológica del paciente diabético, permitiría a los diferentes personajes del equipo de salud institucional contar con la información de utilidad para repercutir positivamente en el control farmacológico de la enfermedad, impedir la presencia de complicaciones agudas, así como retrasar las complicaciones tardías. Un sistema como el propuesto podría además proporcionar información que indague con oportunidad la utilización de servicios de los pacientes diabéticos y que hasta ahora se ha realizado con amplios estudios ${ }^{18}$ donde el nivel de agregación limita distinguir su evolución paulatina dentro de un sistema de salud.

Este sistema propuesto no agota la atención o información del paciente diabético; más aún cuando existen opiniones diversas entre los grupos de expertos (ADA, IMSS, $\mathrm{NOM}^{19}$ ) sobre cuestiones tales como la periodicidad en el control de pruebas de laboratorio, el envío oportuno a un servicio de segundo o tercer nivel, el tipo de diabetes -insulino dependiente o no insulino dependiente-, la patología intercurrente o asociada que complique el cuadro o el pronóstico, el tipo de paciente y la interacción que se establezca entre el médico tratante y el paciente. Tales aspectos han sido considerados de gran complejidad y posibilidades de solución en un estudio que revisó cómo los diabéticos utilizaban un sistema de salud. ${ }^{20}$ Así, este trabajo recoge en una primera etapa los lineamientos básicos de la atención. En una segunda etapa, y ulteriores, se podrán considerar factores como el apego al tratamiento, el conocimiento del diabético acerca de su enfermedad, los factores familiares que apoyan u obstaculizan el manejo y apoyo alimentario o de ejercicio del diabético, etcétera. Es decir, este sistema abre puertas sobre el conocimiento de cómo son, cómo se atienden y cómo responden los pacientes diabéticos a la atención brindada por una institución.

Es pertinente destacar que el diseño de un sistema computacional que organice en forma sistemática la información forma parte de la atención integral al paciente diabético dentro del IMSS, ya que este sistema puede ser aplicado a todos los tipos de diabetes, no es exclusivo del tipo 2, porque los elementos contemplados en el paquete de cómputo (software) corresponden a los lineamientos generales del Programa Institucional. De hecho, el papel dinámico de la monitorización que se propone permite generar los elementos para una evaluación integral del programa, pero también información necesaria para generar investigación clínica, epidemiológica y en sistemas de salud, así como contar con registros clínicos confiables que constituyan una memoria institucional de la atención brindada.

\section{Agradecimientos}

Los autores agradecen a los licenciados en informática Bethsaida Quintero Calva y José Román Pérez Santos por su ayuda en la programación del software; a Augusto Servando Limón Rosas por su valioso apoyo en la captura de los datos; a la trabajadora social Matilde Elena Soto Bermúdez por la asistencia en la 
identificación de los procesos y su colaboración en la recolección de datos, y a los doctores Miguel Bernardo Romero Téllez y Juan Francisco Irízar López por sus valiosos comentarios.

\section{Referencias}

1. D onabedian A. G arantía y monitoría de la calidad de la atención médica. Cuernavaca, Morelos: Instituto N acional de Salud Pública 1992;(Perspectivas en Salud Publica 10):9-12.

2. Donabedian A. Los espacios de la salud: aspectos fundamentales de la organización médica. México, D.F.: Fondo de Cultura Económica 1988;(Biblioteca de la salud), $772 \mathrm{pp}$.

3. 0 rganización Panamericana de la Salud. Sistema de vigilancia epidemiológica de las enfermedades transmisibles y zoonosis.W ashington, D.C.: O PS, 1974:9.

4. O rganización Panamericana de la Salud. Declaración de las A méricas sobre diabetes. W ashington, D.C .: O PS, 1996;D D 39/19.

5. Instituto Mexicano del Seguro Social. Guía técnica general para la vigilancia, prevención y control de la diabetes mellitus. México, D.F.: IMSS, 1999:12.

6. Ruíz-de Chávez M, Molina-Medina H, Cervera-Flores M. Sistemas de información en salud:tendencias actuales. México,D.F.:Secretaria de Salud, 1988:3.

7. C ohen MM, MacW illiam L. Measuring the health of the population. Med Care 1995;33(12): DS21-DS42.

8. Rodrigues RJ. Manual de pautas para el establecimiento de sistemas locales de información.W ashington, D.C.: O rganización Panamericana de la Salud 1996;(Serie HSP-UN I/Manuales 0 perativos Paltex vol. II, 8).

9. American Diabetes Association. Clinical practical recommendations. Diabetes Care 1999;22 suppl 1.

10. Kussman MJ, Goldstein H, Gleason RE. The clinical course of diabetic nephropathy. JAMA 1976;236(16):1861-1863.
11. Pérez-Pastén E, Barrón-U ribe C, Hermida-G utiérrezY. Educación en la diabetes mellitus. En:A riza-Andraca R, Frati-Munari AC, ed. Temas de medicina interna. Diabetes mellitus. México, D.F.: Interamericana, McG raw Hill 1993;1(4):923-934.

12. Secretaría de Salud. N orma 0 ficial Mexicana, N O M-023-SSA 2-1994, para el control, eliminación y erradicación de las enfermedades evitables por vacunación. México, D.F.: Diario 0 ficial de la Federación, DXIV 5, 5 de julio de 1996:21-39.

13. Sepúlveda-A mor J. Epidemiología y vacunación. En: Escobar-G utiérrez A, Valdespino-Gómez JL, Sepúlveda-Amor J, ed. Vacunas, ciencia y salud. México, D.F:: Secretaría de Salud, 1992:3-8.

14. Shlaepfer-D edrazini L, Infante-C astañeda C . La medición en salud; perspectivas teóricas y metodológicas. Salud Publica Mex 1990;32: $141-155$

15. Pogach LM, C utler F, Hawley $G$, Zieve F, W einstock R, Bates $M$ et al. Diabetes prevalence and hospital and pharmacy use in the veterans administration (1994). Use of an ambulatory care pharmacy-derived database. Diabetes Care 1998:21(3):368-373.

16. H ofer TP, Hayward RA, G reenfield S,W agner EH, Kaplan SH, Manning W G. The unreliability of individual physician "Report cards" for assessing the costs and quality of care of a chronic disease. JAMA 1999;281: 2098-2105.

17. C abrera-Pivaral CE, Barba-Borrego JA, Iñiguez-N úñez JE, Ramos-Corrales ], Ramírez-G racia MA, Peña-G uzmán $F$ et al.A mbiente de trabajo en una Unidad de Medicina Familiar. Rev Med Inst Mex Seguro Soc 1994; 32:543-548.

18. González-Villalpando C, Stern MP,Arredondo-Pérez B. Utilización de servicios hospitalarios por pacientes diabéticos; estudio en población abierta. Salud Publica Mex 1994:36:415-419.

19. Secretaría de Salud. N orma 0 ficial Mexicana N O M-015-SSA2-1994 para prevención, tratamiento y control de la diabetes mellitus en la atención primaria. México, D.F.: Diario 0 ficial de la Federación, CDXCV 5,8 de diciembre de 1994:53-67.

20. Engelgau MM, G eiss LS, Mannien D L, 0 rians $C E, W$ agner EH, Friedman $\mathrm{N} M$ et al. Use of services by diabetes patients in managed care organizations: Development of a surveillance system. Diabetes Care 1998; 21(12):2062-2068. 